\title{
Smartphone-Based Human Activity Recognition Using CNN in Frequency Domain
}

\author{
Xiangyu Jiang ${ }^{1}$, Yonggang $\mathrm{Lu}^{1(\bowtie)}$, Zhenyu $\mathrm{Lu}^{1,2}$, and Huiyu Zhou ${ }^{3}$ \\ ${ }^{1}$ School of Information Science and Engineering, Lanzhou University, \\ 730000 Lanzhou, Gansu, China \\ ylu@lzu.edu.cn \\ ${ }^{2}$ College of Computer Science and Engineering, Northwest Normal University, \\ 730070 Lanzhou, Gansu, China \\ 3 Institute of Electronics, Communications and Information Technology, \\ Queens University of Belfast, BT3 9DT Belfast, UK
}

\begin{abstract}
Human activity recognition (HAR) based on smartphone sensors provides an efficient way for studying the connection between human physical activities and health issues. In this paper, three feature sets are involved, including tri-axial angular velocity data collected from gyroscope sensor, triaxial total acceleration data collected from accelerometer sensor, and the estimated tri-axial body acceleration data. The FFT components of the three feature sets are used to divide activities into six types like walking, walking upstairs, walking downstairs, sitting, standing and lying. Two kinds of CNN architectures are designed for HAR. The one is Architecture A in which only one set of features is combined at the first convolution layer; and the other one is Architecture B in which two sets of the features are combined at the first convolution layer. The validation data set is used to automatically determine the iteration number during the training process. It is shown that the performance of Architecture B is better compared to Architecture A. And the Architecture B is further improved by varying the number of the features maps at each convolution layer and the one producing the best result is selected. Compared with five other HAR methods using CNN, the proposed method could achieve a better recognition accuracy of $97.5 \%$ for a UCI HAR dataset.
\end{abstract}

Keywords: Human activity recognition $\cdot$ Convolutional neural network Smartphones $\cdot$ Accelerometer $\cdot$ Gyroscope

\section{Introduction}

With the rapid advancement of technology, smart phones become an integral part of human's daily life. Smartphones are usually embedded with various sensors gathering data for smart security, user authentication, intelligent health monitoring, human activity recognition (HAR) and so on. HAR has been widely used in intelligent wearable device, human computer interaction, athletic training and competition, military, healthcare domains such as health assisted diagnosis and treatment, cognitive disorder recognition systems, elder care support, rehabilitation assistance and so on [1]. 
HAR system is a typical pattern recognition system, which could be divided into several parts, including sensing, segmentation, feature extraction and classification. [2] In the process of the HAR based on smartphones, data collection from built-in sensors is the first step. So, it is very important to choose the proper kinds of sensors in research. Akram Bayat et al. [3] only use acceleration data generated by users' smartphones to recognize six kinds of activities. In the works of Wanmin Wu et al. and Yongjin Kwon et al. [4, 5], accelerometer and gyroscope embedded in a smartphone are both used. They found that combining these two complementary sensors can improve the recognition accuracy. Shinya Matsui et al. [6] use three sensors including accelerometers, magnetometers, and gyroscopes for HAR. Their experiments have proved that different kinds of data from different sensors do improve the recognition accuracy of activities by offering extra information.

The feature extraction in traditional method takes a lot of effort because it has to be done manually. Features extracted in traditional methods can be divided into two types: time-domain features and frequency-domain features. Time-domain features include mean value, standard deviation, kurtosis, skewness, Inter-quartile-Range (IR), correlation between axes, zero crossing rate, etc. Frequency-domain features includes frequency-domain entropy, Fast Fourier Transform (FFT) coefficients and Discrete Cosine Transform (DCT) coefficients, etc. Some others approaches such as Principal Component Analysis (PCA), Autoregressive Model and Haar filters are also used in HAR researches [6, 7].

HAR is a classic multi-classification problem that uses one-dimensional sensor signals and extracts discriminative features to recognize human activities by a classification method [8]. The quality of the classifier has significant influence on the HAR system. Traditional classification methods used in HAR include Support Vector Machines (SVM), k-Nearest Neighbor (k-NN), Decision Tree (DT), Naive Bayesian (NB), Hidden Markov Model (HMM), etc. So, for the traditional methods, it is important to combine an effective feature extraction method with a good classification method in HAR.

Deep learning could transform raw data into more abstract expressions of higher level through some simple but nonlinear models. Complex functions could be also learned by means of enough combinations of transformations [9]. Deep learning carries automatic features extraction instead of manual heuristic operation. That is to say, the core aspect of deep learning is that the features of each layer are not designed for artificial engineering, but rather a universal learning process from the data [9]. For the past several years, deep learning has demonstrated a strong and excellent learning ability in many fields such as computer vision, speech recognition, and machine translation. In the field of HAR, deep learning can enhance HAR efficiency by automatic feature extraction. Convolutional neural network (CNN) is a kind of deep feedforward artificial neural network; it has remarkable performance for large image processing [10-12]. Recently, CNN is also applied to the HAR, and the results are dramatic and encouraging [1, 2, 13-17]. 


\section{Related Works}

The previous works related to HAR using deep learning techniques have shown promising results. Yuqing Chen et al. use a CNN contains 3 convolution layers and 3 pooling layers for HAR [2]. They set the width of convolution kernel to 2 and set up a validation set to locate the best epochs. The average classification accuracy of their method is about $93.8 \%$. A wider time span of temporal local correlation $(1 \times 9$ $1 \times 14)$ with a low pooling size $(1 \times 2-1 \times 3)$ is exploited and is shown to be beneficial to HAR by Charissa Ann Ronao et al. [16]. This work uses the time-series sensor data and additional information of FFT of the HAR data separately as the input to $\mathrm{CNN}$. They show that the accuracy rate reaches $94.79 \%$ for the time-series data, and reaches $95.75 \%$ for the FFT of the HAR data. It can be seen that using the data in frequency domain produces better results than using the data in time domain. Ming Zeng et al. [13] design a simple network architecture, including an input layer, a convolution layer, a max-pooling layer, a 1024 neuron fully-connected layer and a softmax layer to for HAR. Daniele Rav et al. [17] propose a deep learning architecture: a filter is applied to the input, and the weighted sums are computed in the temporal convolution layer which is followed by a fully-connected layer and a soft-max layer for classification. In their works, they decrease the computation cost by limiting the connections from the input nodes in order to extract features efficiently through fewer nodes and levels. The works of Tahmina Zebin et al. show that the performance is noticeably enhanced when the third convolution layer is added [1].

\section{Design of the CNN Architectures}

From the previous works, it can be seen that the $\mathrm{CNN}$ architecture is vital for the final recognition results. So the influence of the network architectures on the recognition accuracy is studied. In the experiments, two different CNN architectures are designed, which are called Architecture A and Architecture B, and they are shown in Figs. 1 and 2 respectively. The differences between the two architectures are the size of input maps and the size of filter maps in the first convolution layer. So the only difference of the two architectures is in the first convolution layer. As shown in Figs. 1 and 2,1-channel and 2D convolution are performed and three convolution layers are applied in our work because the three convolution layers are shown to be a proper setup for HAR $[2,16]$. It is also found in our experiments that using three convolution layers in CNN can produce better results than using one or two convolution layers, while using four convolution layers produces similar results.

The details of the Architecture B are shown in Fig. 1, where the input maps contain tri-axial angular velocity data from the gyroscope sensor, tri-axial total acceleration data from the accelerometer sensor and the tri-axial estimated body acceleration data. The filter size is $13 \times 3$ and the step size is $1 \times 3$.

The details of the Architecture A are shown in Fig. 2. The tri-axial angular velocity data from the gyroscope sensor is duplicated at the end of the input in architecture B for including all pairs of feature sets in the convolution. The filter size is $13 \times 6$ and the step size is also $1 \times 3$. The advantage of doing this is that the information among three 
different sets of features is combined at the first convolution layer: the tri-axial angular velocity information from the gyroscope sensor and the tri-axial total acceleration information from the accelerometer sensor are combined, the tri-axial total acceleration information from the accelerometer sensor and the tri-axial estimated body acceleration information are combined, the tri-axial angular velocity information from the gyroscope sensor and the tri-axial estimated body acceleration information are combined respectively. In this case, each filter map covers two different sensor information in architecture B.

After the input layer, $3 \mathrm{CNN}$ layers are included in the two architectures. Each CNN layer includes convolution, batch normalization, clipped-Relu [18] and max-pooling. After that, dropout, fully connected and soft-max are applied in the two architectures. Then a dropout layer is added to randomly set input elements to zero with a given probability, which is set to $50 \%$ in our experiments. This operation corresponds to temporarily dropping a randomly chosen unit and all its connections from the network during the training. The dropout could prevents overfitting [19, 20].

The validation data set is also used in the experiments to control the number of epochs during the training process. The addition of validation set could prevent the over-fitting of the training.

For different optimizers, many different hyper-parameters are also analyzed in these experiments. In the experiments, the order of the training data is randomized before each training epoch. One common solution is to use a back propagation algorithm to train the network with SGDM [21] and Adam [22].

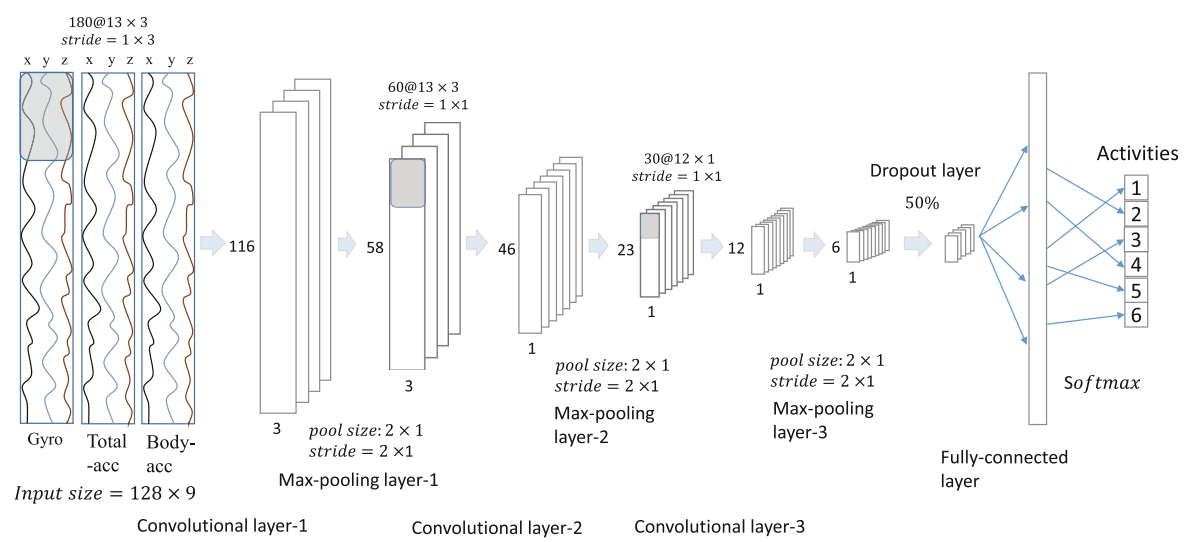

Fig. 1. Deep learning architecture A for HAR. 


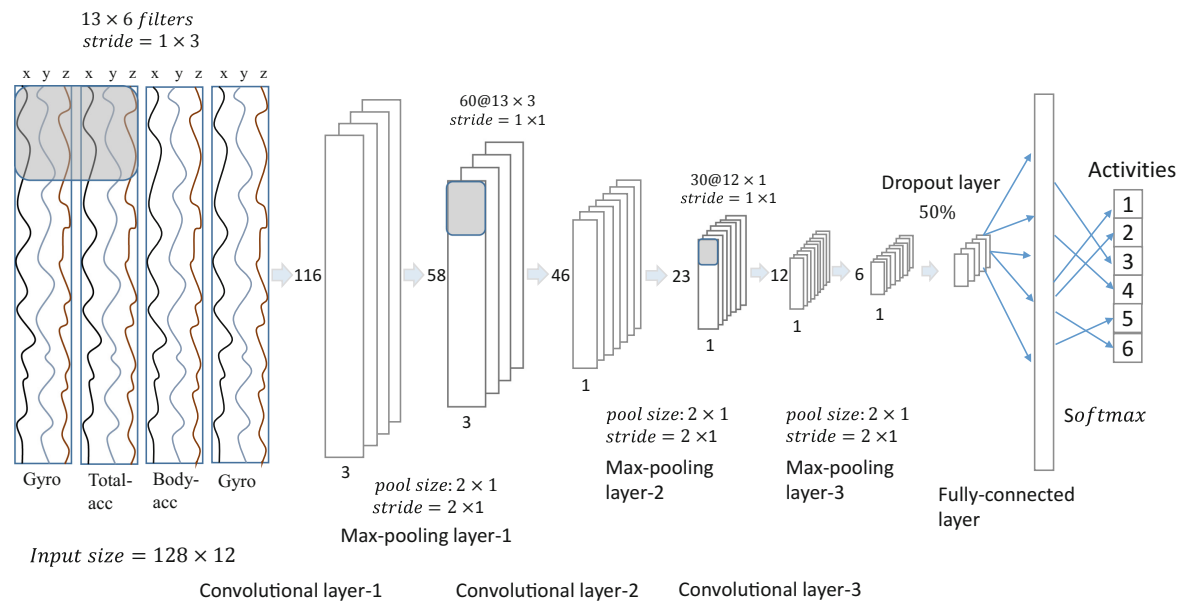

Fig. 2. Deep learning architecture B for HAR.

\section{Experiments}

\subsection{Data Set}

The UCI HAR dataset [23] are downloaded. The UCI dataset includes tri-axial total acceleration data from the accelerometer sensor, the estimated body acceleration and tri-axial angular velocity data from the gyroscope sensor for six kinds of activities including walking, walking upstairs, walking downstairs, sitting, standing and lying. The UCI dataset is collected from a group of 30 volunteers in the 19 to 48 age range. The sensor signal is preprocessed by the noise filter, and then sampled in the fixed width sliding window of $2.56 \mathrm{~s}$ with $50 \%$ overlap. The acceleration sensor signal has gravity and body parts, which are separated by a Butterworth low pass filter to body acceleration and gravity acceleration in the UCI dataset. There are total 7352 examples for the training data and 2947 examples for the test data in the raw date set. For the convenience of calculation, the training set samples is extended to 8000 with random repetition, then 1000 examples are randomly picked from the training set to serve as validation data set, and the test set samples is also extended to 3000 with random repetition. As shown in the previous studies, data in the frequency domain works better for HAR than the data in time domain [24-26]. So only the frequency-domain data is used in our experiments. More specifically, the FFT of the input data are used as the input to the network.

\subsection{Experimental Results}

It is obvious that the choice of hyper-parameters is significant to the performance of the network when $\mathrm{CNN}$ is used to solve practical problems. Therefore, this experiment shows different accuracies with different values of Hyper-parameter settings. 
Validation data set also is applied and the values of two important parameters in validation are evaluated in our experiments. One is the frequency of network validation in terms of the number of epochs, which is set to one. The other is the patience of validation stopping which determine the iteration number automatically during the training process. Figure 3 shows the relationship between the loss on the validation data set and the number of epochs with 30 kernels in each layer and with different optimizers. It is found that 15 is a good setting for the patience of validation stopping. The similar result is found when the number of filter is set to $10,20,30,60,90,120$, 150, 180, 210, 240, 270 and 300 respectively.
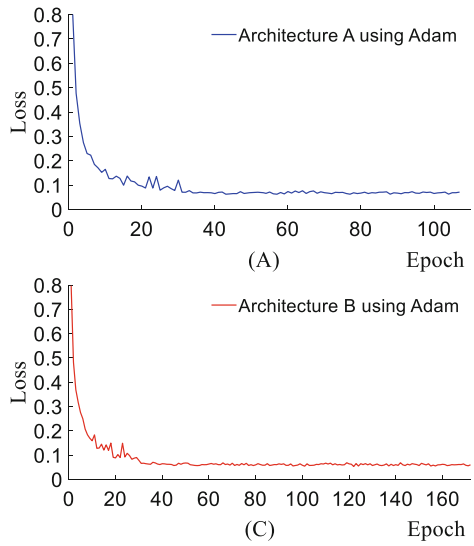
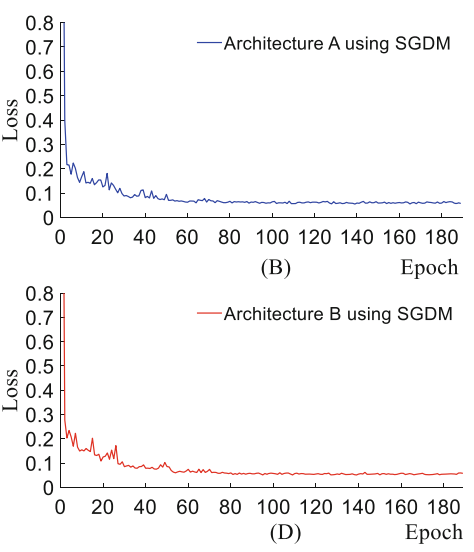

Fig. 3. (A) and (B) are the results for architecture A. (C) and (D) are the results for architecture B. Adam optimizer is used in (A) and (C). SGDM optimizer is used in (B) and (D).

For comparing the two different $\mathrm{CNN}$ architectures, the experiments have been done ten times. For the fairness and objectivity, an accuracy rate is obtained by averaging the results of 10 random experiment results. When the Adam optimizer is used in this CNN architecture, the average accuracy for architecture A is $96.30 \%$ and the average accuracy for architecture B is $96.72 \%$. When the SGDM optimizer is used in this CNN architecture, the average accuracy for architecture $\mathrm{A}$ is $95.91 \%$ and the average accuracy for architecture B is $96.49 \%$.

The results of the 10 experiments are also plotted in Fig. 4. It can be seen that for most cases the final recognition accuracy of architecture B is higher than that of architecture A with both optimizers.

The number of filter maps is very important for the CNN network architectures. For seeking the most appropriate number of filter maps per layer for Architecture B, ten experiments are done with different number of filter maps in different layers. The curves about the average accuracy of the Architecture B under different filter map numbers in the different layers are plotted, where the number of filter maps is set to 10, 20, 30, 60, 

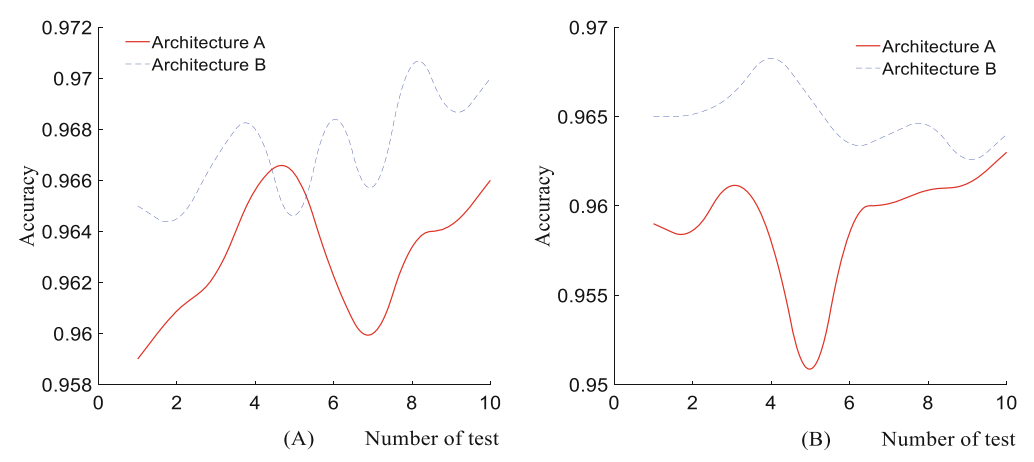

Fig. 4. The result of 10 random tests in the frequency-domain. The Adam optimizer is used in (A) and the SGDM optimizer is used in (B).

$90,120,150,180,210,240,270$ and 300 respectively. First of all, we only change the number of the filter maps in the first layer when all other parameters are fixed. As shown in Fig. 5(A), 180 is the best number for the first layer. Second, we only change the number of the filter maps in the second layer when the number of filter maps in the first layer is set to 180 and other parameters remain the same. As shown in Fig. 5(B), 60 is the best number in the second layer. Finally, we set the number of filter maps in the first and second layer to 180 and 60 respectively, and only change the number of filter maps in the third layer. As shown in Fig. 5(C), 30 is the optimal number of filter maps in third layer. So, a good setting for the numbers of filter maps in the three layers is $(180,60,30)$.

Table 1 shows the confusion matrix produced using the Adam optimizer with the good setting found above for Architecture B. It is found that the recognition accuracy of all six activities is over $93 \%$. And the accuracy of laying is up to $100 \%$, the accuracy of walking and walking upstairs is over $99 \%$. The accuracy of walking downstairs is up to $98.1 \%$. The accuracy of sitting and standing is lower than other activities, but still reaches $95.5 \%$ and $93.3 \%$ respectively. A probable cause is that sitting and standing both are static movements, so the data collected for the two activities are similar, thus the local features extracted by $\mathrm{CNN}$ are similar as well.

The two proposed CNN architectures including Architecture A and Architecture B are also compared with CNN architectures for HAR proposed in other papers for the same UCI HAR dataset. Both Architecture A and Architecture B uses the good setting for the numbers of filter maps which is $(180,60,30)$. The recognition accuracies produced with different CNN architectures are compared in Table 2. From Table 2, it can be seen that using the proposed Architecture B produces the highest recognition accuracy, while using the proposed Architecture A produces the second highest recognition accuracy among all the $7 \mathrm{CNN}$ methods. 


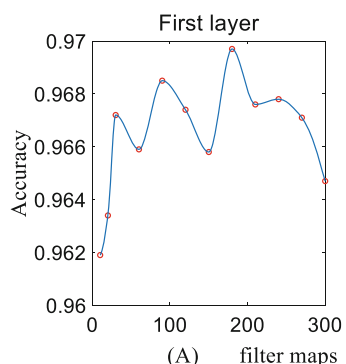

(A) filter maps

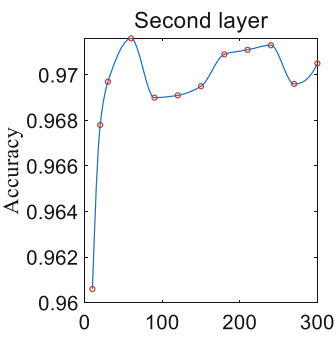

(B) filter maps

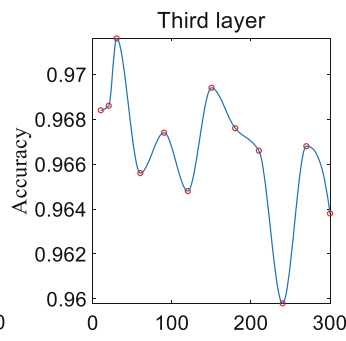

(C) filter maps

Fig. 5. The relationship between the number of filter maps in every layer and the final accuracy of architecture B.

\section{Conclusion}

In this paper, Architecture A and Architecture B are designed for HAR. From the experimental results, it is easily found that the accuracy of the Architecture B is higher than that of Architecture A under the same conditions. It is found that the CNN network architecture which uses the combinations of different kinds of signal sources at the first convolution layer, as in Architecture B, can produce better HAR results. The reason may be that the kind of architecture can extract more discriminative features for classification. Our future work is to apply the Architecture B on more datasets and to improve the recognition rate by adding more features to input data.

Table 1. Confusion matrix of the architecture B

\begin{tabular}{l|l|l|l|l|l|l|l}
\hline \multirow{2}{*}{ Target Class } & Output Class & \multicolumn{4}{ll}{} & \multirow{2}{*}{ Precision } \\
\cline { 2 - 7 } & Walking & Upstairs & Downstairs & Sitting & Standing & Laying & \\
\hline Walking & 500 & 0 & 4 & 0 & 0 & 0 & $99.2 \%$ \\
\hline Upstairs & 1 & 474 & 4 & 0 & 0 & 0 & $99 \%$ \\
\hline Downstairs & 5 & 3 & 423 & 0 & 0 & 0 & $98.1 \%$ \\
\hline Sitting & 0 & 0 & 0 & 462 & 22 & 0 & $95.5 \%$ \\
\hline Standing & 0 & 0 & 2 & 35 & 517 & 0 & $93.3 \%$ \\
\hline Laying & 0 & 0 & 0 & 0 & 0 & 548 & $100 \%$ \\
\hline Recall & $98.8 \%$ & $99.4 \%$ & $97.7 \%$ & $93 \%$ & $95.9 \%$ & $100 \%$ & $97.5 \%$ \\
\hline
\end{tabular}

Table 2. Comparison with other methods

\begin{tabular}{l|l}
\hline Method & Accuracy on test data set \\
\hline CNN [2] & $93.8 \%$ \\
\hline CNN [25] & $95.31 \%$ \\
\hline Deep CNN [1] & $97.01 \%$ \\
\hline tFFT + Convnet [16] & $95.75 \%$ \\
\hline CNN + Stat.features [25] & $96.06 \%$ \\
\hline Proposed Architecture A & $97.08 \%$ \\
\hline Proposed Architecture B & $97.50 \%$ \\
\hline
\end{tabular}


Acknowledgements. This work is supported by the National Key R\&D Program of China (Grants No. 2017YFE0111900).

\section{References}

1. Zebin, T., Scully, P.J., Ozanyan, K.B.: Human activity recognition with inertial sensors using a deep learning approach. In: SENSORS, 2016 IEEE, pp. 1-3. IEEE (2016)

2. Chen, Y., Xue, Y.: A deep learning approach to human activity recognition based on single accelerometer. In: 2015 IEEE International Conference on Systems, Man, and Cybernetics (SMC), pp. 1488-1492. IEEE (2015)

3. Bayat, A., Pomplun, M., Tran, D.A.: A study on human activity recognition using accelerometer data from smartphones. Proc. Comput. Sci. 34, 450-457 (2014)

4. Wu, W., Dasgupta, S., Ramirez, E.E., Peterson, C., Norman, G.J.: Classification accuracies of physical activities using smartphone motion sensors. J. Med. Internet Res. 14 (2012)

5. Kwon, Y., Kang, K., Bae, C.: Unsupervised learning for human activity recognition using smartphone sensors. Expert Syst. Appl. 41, 6067-6074 (2014)

6. Matsui, S., Inoue, N., Akagi, Y., Nagino, G., Shinoda, K.: User adaptation of convolutional neural network for human activity recognition. In: Signal Processing Conference (EUSIPCO), 2017 25th European, pp. 753-757. IEEE (2017)

7. Hanai, Y., Nishimura, J., Kuroda, T.: Haar-like filtering for human activity recognition using 3d accelerometer. In: Digital Signal Processing Workshop and 5th IEEE Signal Processing Education Workshop, 2009. DSP/SPE 2009. IEEE 13th, pp. 675-678. IEEE (2009)

8. Plötz, T., Hammerla, N.Y., Olivier, P.: Feature learning for activity recognition in ubiquitous computing. In: IJCAI Proceedings-International Joint Conference on Artificial Intelligence, pp. 1729 (2011)

9. LeCun, Y., Bengio, Y., Hinton, G.: Deep learning. Nature 521, 436 (2015)

10. Simonyan, K., Zisserman, A.: Very deep convolutional networks for large-scale image recognition. arXiv preprint arXiv:1409.1556 (2014)

11. Sharif Razavian, A., Azizpour, H., Sullivan, J., Carlsson, S.: CNN features off-the-shelf: an astounding baseline for recognition. In: Proceedings of the IEEE Conference on Computer Vision and Pattern Recognition Workshops, pp. 806-813 (2014)

12. An, D.C., Meier, U., Masci, J., Gambardella, L.M., Schmidhuber, J., rgen: Flexible, high performance convolutional neural networks for image classification. In: IJCAI 2011, Proceedings of the International Joint Conference on Artificial Intelligence, Barcelona, Catalonia, Spain, pp. 1237-1242. (2011)

13. Zeng, M., et al.: Convolutional neural networks for human activity recognition using mobile sensors. In: 2014 6th International Conference on Mobile Computing, Applications and Services (MobiCASE), pp. 197-205. IEEE (2014)

14. Wang, J., Chen, Y., Hao, S., Peng, X., Hu, L.: Deep learning for sensor-based activity recognition: a survey. arXiv preprint arXiv:1707.03502 (2017)

15. Hammerla, N.Y., Halloran, S., Ploetz, T.: Deep, convolutional, and recurrent models for human activity recognition using wearables. arXiv preprint arXiv:1604.08880 (2016)

16. Ronao, C.A., Cho, S.-B.: Human activity recognition with smartphone sensors using deep learning neural networks. Expert Syst. Appl. 59, 235-244 (2016)

17. Ravi, D., Wong, C., Lo, B., Yang, G.-Z.: Deep learning for human activity recognition: A resource efficient implementation on low-power devices. In:2016 IEEE 13th International Conference on Wearable and Implantable Body Sensor Networks (BSN), pp. 71-76. IEEE (2016) 
18. Hannun, A., et al.: Deep Speech: scaling up end-to-end speech recognition. Computer Science (2014)

19. Srivastava, N., Hinton, G., Krizhevsky, A., Sutskever, I., Salakhutdinov, R.: Dropout: A simple way to prevent neural networks from overfitting. J. Mach. Learn. Res. 15, 1929-1958 (2014)

20. Krizhevsky, A., Sutskever, I., Hinton, G.E.: Imagenet classification with deep convolutional neural networks. In: Advances in Neural Information Processing Systems, pp. 1097-1105 (2012)

21. Robert, C.: Machine learning, a probabilistic perspective. Taylor \& Francis (2014)

22. Kingma, D.P., Ba, J.: Adam: a method for stochastic optimization. arXiv preprint arXiv: 1412.6980 (2014)

23. http://archive.ics.uci.edu/ml/datasets/Human+Activity+Recognition+Using+Smartphones

24. Huynh, T., Schiele, B.: Analyzing features for activity recognition. In: Proceedings of the 2005 Joint Conference on Smart Objects and Ambient Intelligence: Innovative ContextAware Services: Usages and Technologies, pp. 159-163. ACM (2005)

25. Ignatov, A.: Real-time human activity recognition from accelerometer data using convolutional neural networks. Appl. Soft Comput. 62, 915-922 (2018)

26. Wang, X., Lu, Y., Wang, D., Liu, L., Zhou, H.: Using jaccard distance measure for unsupervised activity recognition with smartphone accelerometers. In: Asia-Pacific Web (APWeb) and Web-Age Information Management (WAIM) Joint Conference on Web and Big Data, pp. 74-83. Springer, Berlin (2017) 